Published in Philosophy of Science 74: 452-480 (2007).

\title{
Mirroring as an A Priori Symmetry
}

\author{
Simon Saunders ${ }^{1}$
}

\begin{abstract}
A relationist will account for the use of 'left' and 'right' in terms of relative orientations, and other properties and relations invariant under mirroring. This analysis will apply whenever mirroring is a symmetry, so it certainly applies to classical mechanics; we argue it applies to any physical theory formulated on a manifold: it is in this sense an a priori symmetry. It should apply in particular to parity-violating theories in quantum mechanics; mirror symmetry is only broken in such theories as a special symmetry.
\end{abstract}

\section{Introduction}

When I say a symmetry of a theory defined on a manifold is a priori, general, or trivial, I mean it in the sense that translations are symmetries of general relativity (GTR) even when spacetime, as defined by the metrical field, lacks translational symmetry. Thus let $g$ represent the metric tensor and $T$ the energymomentum tensor for the non-gravitational fields, defined on a manifold $\mathcal{M}$. For simplicity, let $\mathcal{M}$ be diffeomorphic to $\mathbb{R}^{4}$, so that a single set of rectilinear coordinates $x^{k}: \mathcal{M} \rightarrow \mathbb{R}, k=0,1,2,3$ suffices. Let $t: \mathcal{M} \rightarrow \mathcal{M}$ represent a translation, i.e. for each $q \in \mathcal{M}$, it induces the action $x^{k}(q) \rightarrow x^{k}(t(q))=$ $x^{k}(q)+$ const on the coordinates $x^{k}$. Then, since the field equations of GTR are generally covariant, if $\langle\mathcal{M}, g, T\rangle$ is a model of GTR so is $\left\langle\mathcal{M}, t^{*} g, t^{*} T\right\rangle$, where $t^{*} T, t^{*} g$ are the pull-back of $T$ and $g$ under $t$ (where, in the simplest case of a scalar field, $t^{*} \phi(q)=\phi(t(q))$.)

There are of course infinitely many such symmetries of theories on manifolds; the same is true of any smooth transformation on $\mathcal{M}$. It is trivial translational symmetry, in contrast to the substantive, non-trivial kind, when translations are isometries of the metric, i.e. when they satisfy $t^{*} g=g$. For $t$ an isometry, if $\langle\mathcal{M}, g, T\rangle$ is a spacetime model in GTR, so is $\left\langle\mathcal{M}, g, t^{*} T\right\rangle$. This condition is stringent: it says the metric (and, in GTR, the matter distribution) is perfectly homogeneous. Such symmetries are usually called 'special'.

Whether special or of the trivial, a priori kind, symmetries threaten a form of underdetermination-they yield distinct representations, hence seeming states of affairs, where no observational distinctions are to be found. 'Relationism' as I shall understand it is a general framework for avoiding such underdetermination: it says that only quantities invariant under exact symmetries are

\footnotetext{
${ }^{1}$ To contact the author, please write to: Philosophy Centre, Oxford University, 10 Merton St., Oxford, Oxen OX1 4JJ, UK; e-mail: simon.saunders@philosophy.ox.ac.uk
} 
real-thus relative directions, relative distances, and so on, under rotations and translations etc. A principle like this has been implicit or explicit in most relationist and more generally 'structuralist' interpretations of physical theories, and not only spacetime theories (and not only, for that matter, physical theories, see e.g. Nozick 2001). Call it the invariance principle. The distinctions among representations then correspond to nothing physically real. Equivalently, such models, as goes their physical content, can be simply identified. They are Leibniz equivalent (LE), in the terminology of Earman and Norton (1987).

Every symmetry transformation transforms something relative to something else. In the case of special symmetries certain physical fields $T$ are dragged relative to others $g$, and the existence of the symmetry reflects a symmetry of $T$ or $g$-something that might well be only contingently symmetric. By contrast trivial symmetries signal the symmetry of something much more abstract: symmetries of the bare manifold $\mathcal{M}$, equipped only with its atlas of charts. As such it applies to any theory formulated on a manifold, just as do the diffeomorphism symmetries inherent in the concept of manifold.

I take all of this to be tolerably clear. But its extension, or lack of it, to the discrete symmetries-to permutations and inversions (spatial, temporal, and of matter and antimatter)-is obscure. Here we shall concentrate entirely on spatial inversions in odd dimensions (or mirroring in even ones-we shall use the terms interchangeably). Our claim is that neither mirror-symmetry, nor its violation in quantum mechanics (parity violation), offers any impediment to this program. Invariant quantities (whether under general or special mirror symmetry) are sufficient to account for the appearances and even the workings of theories, here as in the case of continuous symmetries.

To keep things as simple as possible, we shall restrict attention throughout to orientable manifolds. In that case an atlas of charts on $\mathcal{M}$ can be chosen so that every chart is similarly handed (so where charts $x, x^{\prime}$ overlap, the Jacobean of the matrix of derivatives $\partial x^{\prime j} / \partial x^{k}$ is positive). There are two such (diffeomorphism classes of) atlases, call them the left-atlas and the right-atlas. Let $r$ be a manifold homeomorphism inducing the transformation from the left-atlas to the right-atlas and vice versa (see $\S 4$ for a more precise definition). Our claim is that if $\langle\mathcal{M}, g, T\rangle$ is a model of GTR so is $\langle r(\mathcal{M}), g, T\rangle$, and equivalently, so is $\left\langle\mathcal{M}, r^{*} g, r^{*} T\right\rangle$; and that the same is true of any other physical theory formulated on a manifold. By the invariance principle (or LE) the two should be identified as representing the same physical state of affairs.

From a formal point of view that accounts for general mirror symmetry. However special mirror symmetry, and specifically the breaking of this symmetry in particle physics, poses a harder problem: it can hardly be that $T$ cannot be reflected relative to $g$, taking $g$ as the Minkowski space metric, as the latter has special mirror symmetry, so that by trivial mirror symmetry it can. Most of what follows is directed to this question.

To this end we make use of the core relationist treatment of handedness and parity violation to be found in the writings of Earman (1989), Hoefer (2000), Huggett (1999, 2000), and Pooley (2003)), but with departures from their broader perspective, which sees relationism as committed not just to the 
invariance principle of LE but to the non-existence of space, a specifically metaphysical doctrine that may require changes in physics (forcing, say, a Machian theory of gravity, possibly at variance with GTR). Relationism, or structuralism, as we understand it, as imposed by the invariance principle, is in no sense so committed. It is rather a framework for the interpretation of physical theories-it is 'non-reductive relationism', in the terminology of Saunders (2003).

If it is indifferent to one popular context of the debate over relationism, it is sensitive to another: the provision of a uniform approach to the failure of special symmetries for theories on manifolds. The standard device is the relative drag, as already illustrated in the case of translations, where one field is dragged relative to another, yielding a model which is unphysical. But in the face of parity violation in Minkowski space, this cannot be expressed by the relative drag of matter and space, as we have seen. If we are sticking to the manifold formalism, and do not move directly to Hilbert-space structures, defining parity violation in terms of the non-commutativity of the parity operator with the Hamiltonian-a strategy we shall in due course consider-presumably we should find particular handed, material structures, that cannot be inverted relative to each other, as a matter of law.

What are those structures? How might they be used in an epistemic context? (that is, in the context in which the ordinary human hand is used)? But questions like this run against the tenor of conventional relationism, which is intent on denying that chiral standards have any significant intrinsic nature, over and above the fact that they are handed. Thus Pooley (2003, 259-60) holds that the only thing that distinguishes a standard for 'left' from other handed objects is the fact that it is called 'left'. He gives the example of proper names: no more is there anything about Immanuel Kant that made it correct to call him 'Immanuel Kant' other than that he was called 'Immanuel Kant'. That is I think the right analysis of how ordinary talk about handedness gets off the ground, but we are interested in something else: what might count as a standard for 'left' that can be used with modal force. A concern of this kind has been expressed before (by Huggett [2000, 235] and Hoefer [2000, 253]), but to a different purpose-the question of how the parity violating laws are to be defined as having one sense, rather than the other. This question, we shall eventually conclude, is entirely empty.

The idea of a standard that can be used in modal contexts deserves some new terminology. A standard is internal if it is modelled explicitly, and external otherwise. Evidently the mere fact that a system is modelled in relation to others does not give those relations a modal force of the sort we are looking for (these relations may be entirely contingent), and to mark this point we shall sometimes speak of a dynamical internal standard; but in principle to be modelled at all is to be subject to physical laws. We are not interested in 'purely kinematic' models (if there are such; see Brown [2005]).

The distinction between 'internal' and 'external' standard (and, by extension, determination) is to be sharply distinguished from that between an 'intrinsic' and 'extrinsic' standard or determination, which is widely in use in the literature on handedness. It is much closer to Stachel's (1993) distinction be- 
tween 'nondynamical' and 'dynamical' determinations, or 'individuating fields' (in spacetime theories), and to Bartlett et al.'s (2005) distinction between 'nonimplicated' and 'implicated' coordinates (in quantum mechanics). See Saunders (2003) for further discussion of Stachel's distinction.

From our point of view the use of an external standard is essentially a copout. An external (nondynamical, non-implicated) standard has to come from somewhere, a supposed background physical system not itself modeled explicitly. Such a background system is always available, as long as the model is restricted to a subsystem of the universe, and the symmetry, or lack of it, is then a subsystem symmetry (in the terminology of Brown and Sypel [1986]). But that is problematic in cosmology; 'relationism' in our sense may not be reductionism (about space) but it is certainly a response to the underdetermination questions that preoccupied Leibniz in his correspondence with Clarke. These questions were always cosmological in scope. Correspondingly, the symmetries of relevance to us are always global transformations of closed physical systems (and the invariance principle and LE apply only to these).

Our program, in summary, is this: when mirroring is not a special symmetry, to give an internal, dynamical model of handed systems, the relative reflection of which is dynamically excluded. First, however, we consider the situation in classical mechanics, where mirroring is a special symmetry.

\section{Mirror Symmetry in Classical Mechanics}

It seems obvious that if a configuration of particles is handed, it must be handed in one way or the other, which way being reversed on mirroring. It is further clear that mirroring is a symmetry of classical mechanical theories. In such theories, then:

\section{Mirroring is a symmetry.}

2. A handed configuration of particles or fields is either left-handed or righthanded and is reversed by mirroring.

By the invariance principle it follows from (1) and (2) that e.g. 'being lefthanded' is not a real physical property, an apparent absurdity. In terms of LE, it follows from (1) and (2) that the world and its mirror image are the same, and hence that left-handed and right-handed things are the same, again an apparent absurdity.

This puzzle dates back to Kant's argument from incongruent counterparts that was in turn inspired by the arguments of the Leibniz-Clarke correspondence. The great difference is that whereas in the case of translations or rotations a given state of affairs and its translate or rotate will at least look just the same, when considered in themselves, that is no longer true when it comes to mirroring. 


\subsection{Global and subsystem mirroring}

(2) can hardly be faulted when mirroring is a subsystem symmetry, when only a subsystem is subject to a symmetry transformation (under an 'active' interpretation-as when Galileo's ship is put out to sea), but it is in question when everything is mirrored. For consider:

(i) Let everything and everyone, including language users, be mirrored. Since it is a symmetry, the result will be physically possible if the original was. But the linguistic, neurological behaviour in this mirror-image world will be functionally the same. Differences, if there are any, will be inexpressible.

My doppelganger's speech patterns, as longitudinal vibrations in the air, are the mirror-images of mine, but that does not make for a difference in what he says. Nor, if inexpressible, should we admit there is a difference in the two cases in what he perceives or in what he believes. It is independently implausible that because his neural processes are the mirror images of mine (and are otherwise identical) that he will perceive things differently from me or understand things differently from me. The two worlds will be indiscernible from within. (An argument of this sort was first made by Earman [1989, 145-46].)

The same doubt can be raised independent of thought or language:

(ii) The Cinderella experiment: given a handed object, and an experiment that determines it to be 'left', the mirror-image experiment will determine the mirror-image object to be 'left' as well.

The apparatus incorporates a handed object that can be compared with the object to be tested (say a shoe, or a glove, or a screw). Thus if the shoe fits, a bell is rung. A foot is presented and the bell is rung. Now consider the mirrorimage of this apparatus, presented with the mirror-image foot. Still the bell is rung! Orientation as measured by a Cinderella experiment is invariant under global mirroring.

These arguments raise doubts as to whether 'left' and 'right' can really differ intrinsically at all. For further well-known arguments to this end, that bring in the dependence of the orientation of a body on the dimensionality or orientability of the space in which it is embedded, see Pooley (2003 p.252-62). On this basis we can simply reject (2). But there is a prior question here: of course there are standards for the correct use of 'left' and 'right', which advert to concrete handed systems. What systems, exactly? What is the Cinderella apparatus, to put the matter in operational terms? It will include a handed structure for comparison with others (by relations invariant under mirroring), but it must include something else; the buck has to stop somewhere.

This 'something else' cannot of course reside in being handed in one particular way rather than the other, since 'being handed in one particular way' is not invariant under mirroring, and hence, by the invariance principle, is not a physically real property. We conclude that it has some distinguishing, invariantly 
described, and therefore nongeometric property (or if it is a geometric property, it is one that is invariant under mirroring).

Before considering in more detail what it is, it is worth saying that the relationist of my stripe need not be committed to denying that 'left' and 'right' are genuinely meaningful as purely mathematical concepts. Platonists may well maintain that a right-handed Cartesian coordinate system is a perfectly good abstract singular object. And unlike the case of the continuous symmetries, it is rather more plausible to suppose one has a direct intuition in this case: a state of rest, in Platonic heaven, is hard to take seriously, and impossible to make use of, but left-handed coordinates seem to be there for the taking and available for inspection in the mind's eye.

We should grant the possibility rather than squash it outright. By all means, suppose a left-handed atlas of charts exists in an abstract sense; suppose even that it can be an object of singular thought. The isomorphism claim-that any mathematical structure built up from the left-hand atlas can be built up from the right-hand atlas as well-is an entirely independent matter. In no sense need the invariance principle (or LE) apply to abstract mathematical objects. But the one condition we do insist on is this: any such perception of abstract mathematical objects is to be sharply distinguished from any visual, sensible perception of physical objects. The point is not that there must be an indivisible gap between the abstract and concrete (that we can leave as an open question), but that we have already taken care of sensible perception of physical objects: they, and if necessary the observer, are to be modeled explicitly (the object as a standard is an internal, embedded standard).

\subsection{Left and right as internal standards}

To summarize, the relationist needs only to reconcile

MS1 Mirroring is a global symmetry

which rules out the use of an external standard, with:

MS2 A handed object is determinately (measurably) either left-handed or righthanded, and is invariant under global mirroring.

We may break (MS2) into two parts. First, purely geometric determinations of relations of congruence and incongruence among handed objects (for convenience, call 'enantiomers'). This is unproblematic in the case of an orientable space (and the ambiguities introduced in a non-orientable space are easily removed):

MS2a An enatiomer is left-handed or right-handed as determined by its congruence and incongruence relations with other enantiomers.

Such determinations are clearly invariant under mirroring.

However the buck must stop somewhere. (MS2) also involves: 
MS2b Certain enantiomers are determined as left-handed, and others right-handed, by virtue of some nongeometrical feature of such objects.

With that, so long as the 'certain standards' mentioned in (MS2b) are incorporated into the physical description, one has a characterization of 'left' and 'right' as internal determinations.

According to Pooley, all that is at issue with (MS2b) is use of language. The additional non-geometric feature of such objects is just that they reliably get called 'left' or 'right', on what basis no matter. And if one looks at how the use of 'left' and 'right' is actually taught, it would seem that any system of rules would do, linking certain sorts of handed objects with some standard; all that would be needed of the standard over and above the fact that it is handed is that (as a purely practical matter) it had no incongruent counterpart, or if it does have an incongruent counterpart, that it be recognizably distinct from it (say, that it be differently colored). In practise, the human hand on the side of the heart does very well (since most human hearts are on the same side of the body); but so would the side of a car near the steering wheel, at least in certain countries; the side of the pedals furthest from the accelerator, in all countries. It would be absurd to single out any one of these features (all invariantly described) as the feature that makes its bearer left-handed. It could even be a certain muscular twitch that I use to tell me which of my hands is right-meaning, which is the one I have been taught to call right, in conformity with others' use of the term.

With all of this we should agree, as goes our ordinary use of the terms 'left', 'right'. It is certainly consistent with the invariance principle: whatever these criteria, they are invariant under global mirror imaging. Nor, up to this point, is the situation really so unfamiliar. Consider the case of length. Time was when the definition of length in SI units was given by congruence relations with a particular rigid object, maintained under controlled conditions in a definite place (a platinum bar, located in a vault in Paris). We may imagine, in the same vault, a particular glove as well (say, a boxing glove, that cannot be turned inside out). To be one metre long just is to have the same length as that particular bar (picked out by features independent of its length). Is our proposal not just the same? Even the obvious difference with length, that there is in fact no need for any physical glove, is easily explained: chiral relations are 2-valued, unlike relative distances, so there is no need for precision; they can be told at a glance; is there any more to the difference between chiral and distance relations than this?

Suppose, indeed, that only the orders of magnitude of relative lengths were ever required: then there would be no need of the platinum rule either. And in practise that is what we still do, making do with the foot, the arm's length, and the finger's breadth, relative dimensions that can be judged at a glance. Telling the approximate height of a man in feet is no different from telling his left hand from his right. It is the same with all other familiar determinations, for weight, temperature, colour and smell: they all begin as qualitative judgments. The difference, on this line of thought, is only between judgments that allow 
for complex logical comparisons (geometry and number), from those that don't (colours and smells).

All this fits with the suggestion, in the case of mirror-symmetry, that the standard is ultimately singled out on the basis of some nongeometric quantity; and with the suggestion, further, that this nongeometrical property can be entirely arbitrary. Thus the particular bar of platinum that was used as a standard of length was essentially chosen arbitrarily. When it comes to the choice of a standard glove to put alongside it, that too could be wholly arbitrary. But it is equally clear that these standards, when modeled as internal to the total system, do not exactly lend themselves to this task. Platinum bars and boxing gloves are hardly natural systems to include in a dynamical model of an atom, or a fluid, or a crystal. Just for that reason, no macroscopic bar could ever provide a very precise standard that could actually be used-and the platinum bar did not long stay the standard.

A first step in improving the accuracy of length determinations was to control for fluctuations in its length (of how it was mounted and at what temperature and pressure, controlled in what way). This was to some extent a matter of modelling the bar dynamically, but proved a complicated matter, insufficiently accurate, not only in controlling for variations in the bar itself but in using it to calibrate other rules. In 1960 the SI unit of length was changed to a certain number of wavelengths of an emission line of Krypton 86, a standard that could be used to calibrate other emission lines very easily. ${ }^{2}$ There remained non-geometrical features to it of course-the particular isotope and the particular atomic transition process- as well as purely numerical conventions (the number of wavelengths chosen), but fix on the latter and the standard has a clear dynamical characterization (the minimal theory to do this in this case is quantum electrodynamics [QED]).

Is it a stretch to similarly seek an internal, dynamical standard in the case of the discrete units-say a sample of positive (rather than negative) charge, as we have a sample of left-handed (rather than right-handed) hands? Surely not, if we include time-inversion along with mirroring and charge-conjugation, the family to which it belongs. The goal of finding an internal, dynamical standard of the forward (rather than backward) arrow of time is widely shared.

The obstacle to defining internal standards in simple dynamical terms, in the case of the discrete symmetries, is not that they are not needed but that they seem impossible to find. There seems to be no simple classical mechanical model of 'the positive ' (charge) or 'the left' (handedness) or for that matter of 'the forward direction in time'. Worse, there may be no complicated classical model either, no internal dynamical standard of any description. For how can any of those things (the positive, the left-hand, the future arrow) be dynamically characterized, if on inversion the dynamics is the same? Just here there is an important contrast with the case of length: there is indeed a dynamical, internal standard of length, in QED, that can couple to other physical systems, but QED

\footnotetext{
${ }^{2}$ It was changed again in 1983, when it was subordinated to a definition of the second (and defined in tems of the distance travelled by light in a certain fraction of a second).
} 
does not have scaling as a special symmetry.

The implication, restricted to mirror-symmetry, seems to be clear. The relationist strategy, in the face of underdetermination with respect to mirroring, is to identify a model and its mirror-image just as it identifies models related by continuous symmetries. Invariant quantities alone are shown to be sufficient to characterize a certain class of things as left-handed, on the basis of their intrinsic properties. The method works because of a trick-these internal properties are independent of geometry per se. But the price is that they appear wholly arbitrary; they are whatever language use happened to most conveniently fix onto. Just insofar as mirroring is a symmetry, it seems the internal standard cannot be characterized dynamically.

\section{Parity violation}

The situation is transformed in moving to a parity-violating theory, like the electroweak theory in quantum mechanics. The way is then open to defining a chiral standard that is not just internal, but non-arbitrary too-a genuinely dynamical marker. But then, if mirroring isn't a symmetry, why isn't the entire relationist program simply an irrelevancy? For if mirroring is no longer a symmetry there is no longer a threat of underdetermination.

The answer is that mirror symmetry remains as an a priori symmetry, and poses just as great a threat of underdetermination in that guise. Nothing in the previous section (except at the very end) hinged on the difference between special and a priori or trivial mirror symmetry.

To begin, we should learn how parity violation is experimentally manifested, and how it is represented in the relevant equations (quantum electroweak theory or QET). This ground has been covered by Earman (1989) and in more detail by Pooley (2003), but their basic points need repeating and, in one instance (the definition of a spin structure on a manifold) they need to be extended.

\subsection{Evidence for parity violation}

We follow Earman in considering the experiment of Crawford et al. (1957). It was observed that when a negative pi meson, $\pi^{-}$, scatters off a proton, at certain energies it decays into two neutral particles (the hyperon, $\Lambda^{0}$, and the neutral $K$-meson, $K^{0}$ ), defining a plane. The hyperon subsequently decays into another pi meson $\pi^{-}$and a proton, which lie on (in general) a different plane. Now take the mirror image of this process through the first of the two planes, so whereas before, e.g., if the final $\pi^{-}$particle moved out of the plane going down, in the mirror-image process, it moves out of the plane going up. It turns out that processes of the second sort are much rarer than processes of the first sort (see Figure1).

Figure 1.-Parity violation for pi meson decay. 


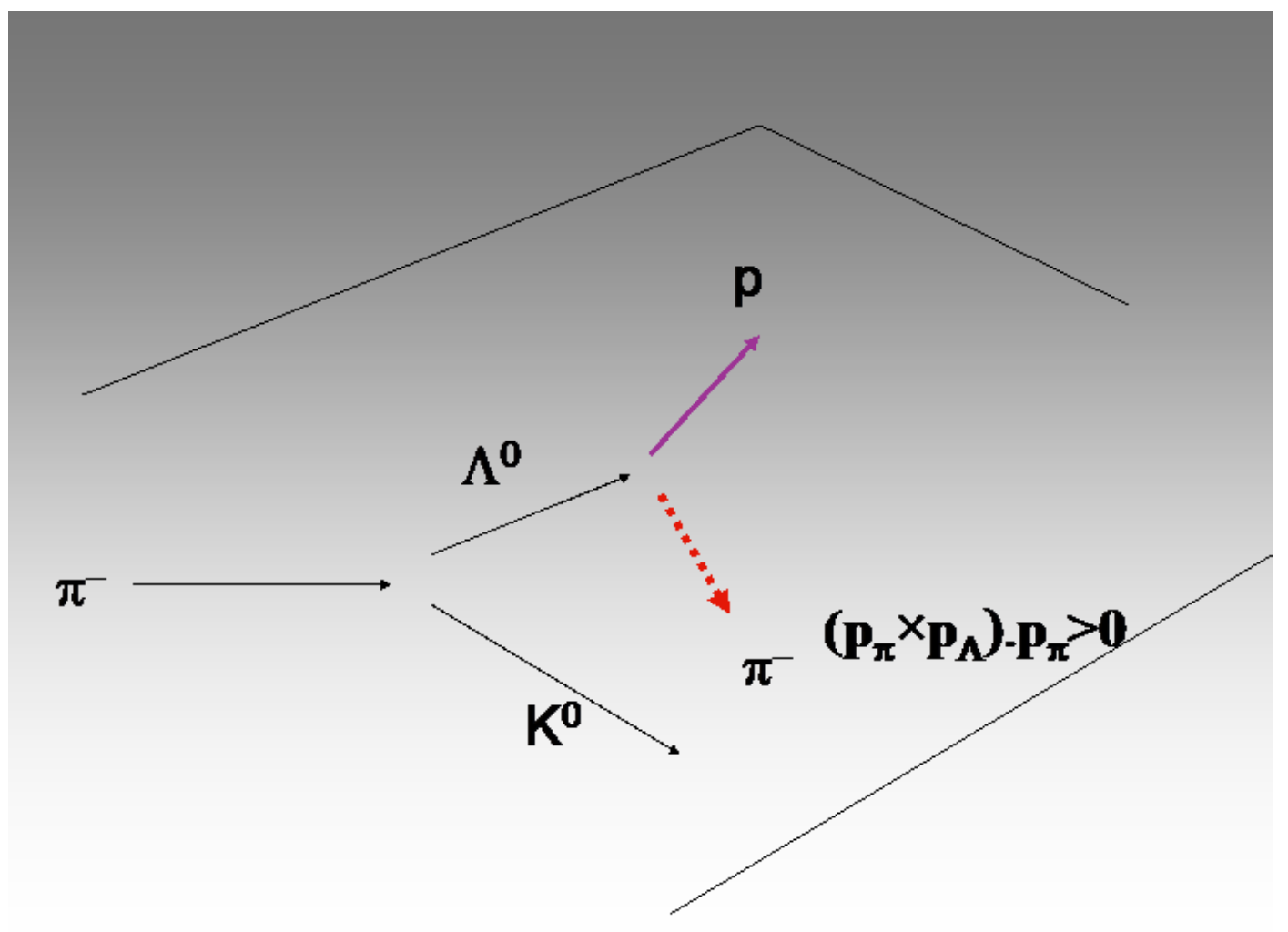




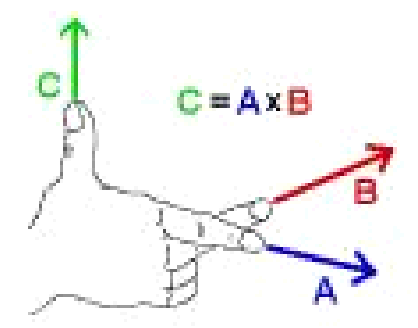

We may quantify the result in terms of the cross product $\overrightarrow{\vec{\pi}_{i n}} \times \overrightarrow{p_{\Lambda}}$ of the momentum vectors of the hyperon and the pion present before the second decay. Let the momentum vector of the final pion be $\overrightarrow{p_{\pi_{f i}}}$. Then the two processes, the one the mirror-image of the other, can each be defined as a condition on the pseudoscalars

$$
\begin{aligned}
& \left(\overrightarrow{p_{\pi_{i n}}} \times \overrightarrow{p_{\Lambda}}\right) \cdot \overrightarrow{p_{\pi_{f i}}}>0 \\
& \left(\overrightarrow{p_{\pi_{i n}}} \times \overrightarrow{p_{\Lambda}}\right) \cdot \overrightarrow{p_{\pi_{f i}}}<0
\end{aligned}
$$

Since this is quantum mechanics, the effect is only statistical, but nevertheless real: one of them (the positive value) is preferentially observed over the other (so [1a] is the correct equation for this kind of decay). Correspondingly, it is not preserved under mirroring (which interchanges [1a] and [1b]).

The cross-product in turn is normally defined, in elementary texts, with the aid of a hand-rule, either explicitly using one or other of the small family of chiral terms, 'clockwise', 'screw', 'right-hand', or by means of a diagram. Quantitatively, if $\theta$ is the angle between two vectors $\vec{A}, \vec{B}$, of magnitude $A$ and $B$ respectively, $\vec{A} \times \vec{B}$ is a vector of magnitude $A B \sin \theta$, orthogonal to the plane defined by $\vec{A}$ and $\vec{B}$. It's direction is fixed by Figure 2 . This picture is not itself modelled in the equations - it is an external standard.

Figure 2.-The right-hand rule.

Insofar as the phenomenological equations go, and in agreement with the account of $\S 2$, 'left' and 'right' are being used as external determinations. This is consistent with taking mirror symmetry as a subsystem symmetry, and its violation as the violation of a subsystem symmetry.

\subsection{Electroweak theory}

In a certain sense, in quantum mechanics, any application of the theory, any symmetry, can only be a subsystem symmetry, for the theory requires an outside observer or experimental context that is not itself a part of the system that is 
modelled. Quantum states $\psi$ are only attributed to subsystems, and their unitary evolutions under Hamiltonians $H$ are only applied to subsystems. There is no 'wave-function to the universe': the observer, in quantum mechanics, cannot be 'naturalized'.

All of these statements are of course highly contentious-the 'certain sense' was Bohr's view of the matter. The three leading realist alternatives, hiddenvariable theories, dynamical collapse theories, and the Everett interpretation (where only the latter leaves the equations of quantum mechanics unchanged), do suppose there is a wave-function for the universe and are perfectly able to naturalize 'the observer'. But for the moment we are asking about the equations of QET as ordinarily applied to parity violating systems, which are undoubtedly modelled as sub-systems of the universe. We are talking of the violation of a sub-system symmetry.

As ordinarily applied, the condition for mirroring to be a symmetry in quantum mechanics is that the Hamiltonian, $H$, commute with the inversion operator, $P$ (a unitary operator); it is broken as a symmetry if $[P, H] \neq 0$. This means that if $\psi(t)$ is a solution to the Schrödinger equation with the Hamiltonian $H$, then $P \psi(t)$, is not such a solution, and does not represent a physically possible evolution of the subsystem.

It seems we have everything we desire, save for the fact that these states of affairs (or disallowed states of affairs) are defined relative to a given background, the classical world, classically described experiments, which are all external standards. The analysis is immediately problematic as soon as we insist, as we must, that quantum mechanics be applied to a strictly closed system (with the observer modelled as within). This, the direct route, is hamstrung by questions in the foundations of quantum mechanics, not least the problem of measurement.

But it appears that for present purposes we can sidestep these questions. We can go a considerable way to understanding the nature of parity violation by considering the structure of the parity-violating Lagrangian as a system of fields (quantum or classical) defined on a manifold, to the neglect of the state. (In field theory one anyway works in the Heisenberg picture, in which the state, independent of measurement, does not change.)

In this spirit, consider the idealized case in which all the fermions are treated as massless. The parity violating part of the interaction Lagrangian is ${ }^{3}$ :

$$
\overline{E_{L}}(i D) E_{L}=\overline{E_{L}} i \gamma^{\mu}\left(\partial_{\mu}-i g A_{\mu}^{a} \tau^{a}-\frac{i}{6} g^{\prime} B_{\mu}\right) E_{L}
$$

This is a Lorentz invariant, $U(1)$ and $S U(2)$ gauge invariant, self-adjoint scalar. $D$ is the covariant derivative; it is a rule to define a derivative, by which one can compare the phase of the various fields at different points in space. Since the rule is path-dependent, or non-integrable, it introduces gauge fields into the theory (the fields $A_{\mu}^{a}, B_{\mu}$ ). Here $B_{\mu}$ for $\mu=0,1,2,3$ are the components of a $U(1)$ gauge field (that enter into the photon coupling as well as that of

\footnotetext{
${ }^{3}$ My notation follows Peskin and Schroeder (1995 equation 20.78), except that I have omitted the right-handed chiral fields (which are present in QED and QCD, but not in EQT, with the same couplings as their left-handed partners to the gauge fields).
} 
the neutral vector boson $\left.W^{0}\right)$ and $A_{\mu}^{a}$ those of an $S U(2)$ gauge field symmetry (one vector field for each of its generators, and two of the three associated with the charged vector bosons, the $W^{ \pm}$particles). $E_{L}$ is the left-handed fermion doublet $\left(\nu_{e}, e^{-}\right)_{L}$, where $\nu_{e}$ and $e^{-}$are the neutrino and electron spinor fields. Because massless, the fermion doublet has a unique frame-independent helicity, which we use to label it as ' $L$ ' or ' $R$ ' respectively. Only if a theory contains both kinds of fermions (or fermion doublets) can it host a representation of the full Poincaré (or 'improper' Lorentz) group, which includes the inversions.

The absence of right-handed fermions from this theory shows rather vividly that it violates mirror symmetry, as the action of the inversion operator $P$ is to interchange them. In fact, it is sufficient (and this is how it works in more realistic models) for the two chiral spinor doublets to couple to gauge fields of different symmetries, or to inequivalent representations of the same symmetry. The kinetic energy term for Dirac fermions $\psi$ can always be split into separate pieces for the left- and right-handed fields:

$$
\bar{\psi} i \gamma^{\mu} \partial_{\mu} \psi=\bar{\psi}_{L} i \gamma^{\mu} \partial_{\mu} \psi_{L}+\bar{\psi}_{R} i \gamma^{\mu} \partial_{\mu} \psi_{R}
$$

The derivatives can then be replaced by covariant derivatives using different gauge groups or different representations of the same gauge group. The result will be a $P$-violating theory.

The key question concerns the use of 'left' and 'right' as designations of the spinor fields. They have a clear geometric meaning in terms of helicities-the projection of the component of spin in the direction of motion-but a meaning fixed by the use of a hand rule, either the left-hand rule or the right. Do they have an independent algebraic significance? As Dirac bi-spinors they are defined by the projections

$$
P_{ \pm}=1 \pm \gamma^{5}=1 \pm \gamma^{0} \gamma^{1} \gamma^{2} \gamma^{3}
$$

where $P_{+} \psi=\psi_{L}, P_{-} \psi=\psi_{R}$. The Dirac $\gamma$-matrices are $4 \times 4$ matrices that transform as a Lorentz vector and obey the anticommutation relations:

$$
\gamma^{\mu} \gamma^{\nu}+\gamma^{\nu} \gamma^{\mu}=2 g^{\mu \nu}
$$

The change from a left-handed to a right-handed coordinate system interchanges two of the $\gamma$-matrices (for $\mu, \nu \in\{1,2,3\}$ ), and therefore interchanges $P_{ \pm}$and $P_{\mp}$. The same can be achieved by a change of representation of these matrices, consistent with their transformation properties under the Lorentz group and the algebra of (4).

The same point can be made in terms of two-component spinors. Quite generally, when introducing spinors on a manifold, one needs a spin structure-a correspondence between tensors acting in the tangent space $T_{q}$ at each point $q \in \mathcal{M}$ and endormorphisms acting on a complex vector space $V_{q}$ at $q$-linear transformations of spinors at $q$. In the case of the Lorentz group, the fundamental connection is between a second order Hermitian spinor field $\alpha_{\dot{A} B}$ (dotted indices are complex conjugates of undotted ones), acting on a 4-dimensional 
complex space and a real vector field, $A$, on Minkowski space, as given by four real functions $A_{\mu}, \mu=0,1,2,3$, thus:

$$
\alpha_{\dot{A} B}=\sigma_{\dot{A} B}^{\mu} A_{\mu} .
$$

Here the four matrices $\sigma_{\dot{A} B}^{\mu}$ are Hermitian and transform as a second-order spinor in the indices $A, B$ and as a Lorentz four-vector in the index $\mu$ (my notation is standard, following e.g. Davis [1970, 281-83]). These matrices are, of course, the Pauli spin matrices (with the $\mu=0$ matrix given by the identity), where their spin indices refer to their transformation properties under $S L(2, C)$, the unimodular group of complex linear transformations in 2 dimensions, the covering group of the proper Lorentz group.

The Pauli matrices, just like the $\gamma$ matrices, anticommute for $\mu \neq v$; mirroring interchanges two of them with a corresponding change in sign. The choice of one sign rather than the other indicates one of the two spinor representations (that are interchanged under mirroring) one is using. Thus, for the free zero-mass neutrino field, one has the two possibilities:

$$
\begin{aligned}
& \left(\sigma_{\dot{A} B}^{0} \partial_{0}-\sigma_{\dot{A} B}^{k} \partial_{k}\right) \nu_{L}^{B}=0 \\
& \left(\sigma_{\dot{A} B}^{0} \partial_{0}+\sigma_{\dot{A} B}^{k} \partial_{k}\right) \nu_{R}^{B}=0
\end{aligned}
$$

(together with the complex conjugate equations describing their antiparticles), corresponding to massless left-handed and right-handed neutrinos respectively. It is only when there are pairs of spinor fields of the same mass that one can define a representation of the full Lorentz group (as with Dirac bispinors).

Which of $(6 \mathrm{a}),(6 \mathrm{~b})$ is the right one? The one that gives the right sign for the measured pseudoscalars -the one that gives (1a) rather than (1b), where the correctness of the latter is determined by a hand-rule, Figure 2. That is essentially to define the spin-structure itself in terms of this hand-rule, just as we did the cross-product. Once this has been done, one can predict the orientation of every other parity-violating decay process in the scope of the theory. Had they been equipped with it in advance, Crawford and his collaborators would have been able to predict which laboratory wall the decay products were headed for.

But just because the choice of sign is made in this way, it is clear that 'left' and 'right' are being used as external determinations. The external standard (human hands and whatnot) is not itself described by the equations. Rather, the external standard is used to pick out one of two sets of equations, that are mapped into one another under mirroring. The situation in these regards is no different from the phenomenological equations $(1 \mathrm{a}, 1 \mathrm{~b})$.

\subsection{The invisibility of mirroring as an a priori symmetry}

Evidently we need to go beyond mirror symmetry breaking as defined by an external standard, and mirror symmetry as a subsystem symmetry. How, ex-

actly? Obviously, by modeling these external standards explicitly. But once 
product

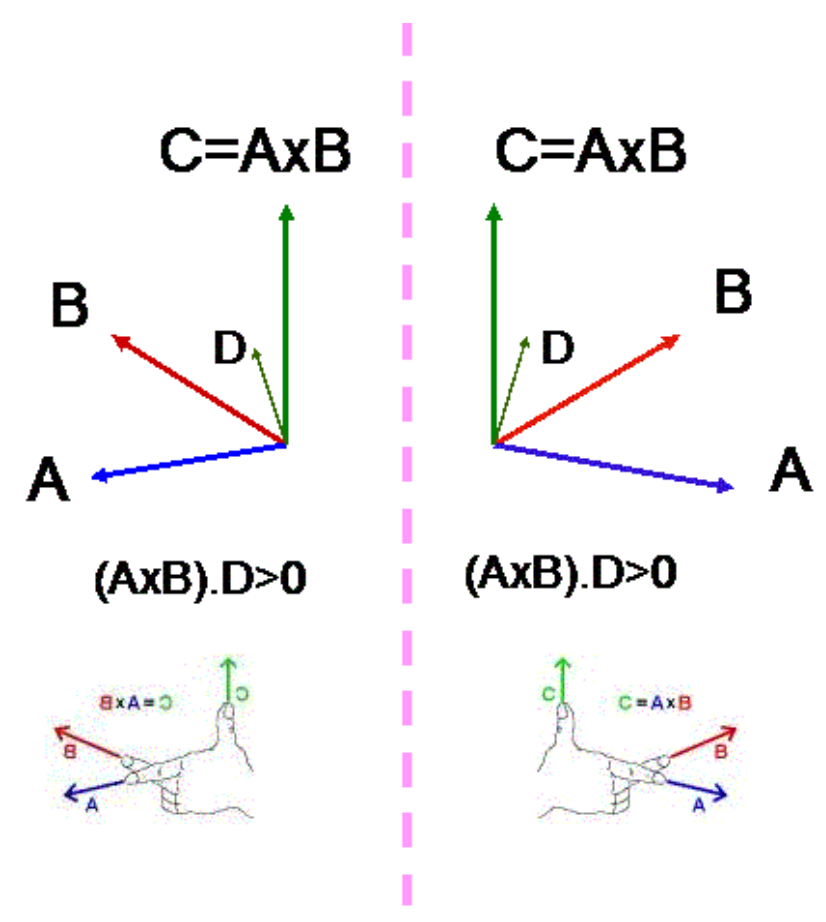

$3 . p d f$

this is done we expect, from the Cinderella experiment, that global mirroring (including mirroring of the standard) will become invisible. Pseudoscalars will no longer be measurable.

To rehearse the steps of the argument, but applied to a parity-violating experiment, consider the phenomenological equations (1a), (1b). They are given a definite sense by an external standard, Figure 2. If the hand depicted were included in the phenomenological description of the decay product, the description would be correct insofar as $A$ is the pion direction, $B$ the meson, and $C$ the kaon, and the fingers and thumb of the hand can be correctly aligned with the decay products, as in Figure 3(a). But now if everything is mirrored that alignment will remain correct, as shown in Figure 3(b). There can be no possible evidence internal to worlds that could tell us which of the two worlds is ours. Correspondingly, the phenomenological equation (1a) becomes invariant under mirroring.

(b)

(a)

Figure 3.-Invisibility of global mirroring as a trivial symmetry. 
What has just been demonstrated is the invisibility of global mirroring as a trivial symmetry, where everything is mirrored relative to the atlas of charts, including what was hitherto used as an external standard.

All well and good, but if we are after a statement of the violation of special mirror symmetry, we should seek a standard whose relative drag, leaving fixed the QET matter and gauge fields of $\S 3.2$, yields a model that is not physically possible: for this purpose human hands will hardly do. Indeed, from a relationist point of view, the contingency of any particular hand rule is of the sort: the hand on the side of the typical human heart is congruent to certain decay processes.

Not only is that a bad candidate for a dynamical internal standard, it is not even clear that the notion of its relative drag is even well-posed. For no hand, or anything else made up out of ordinary matter, could be spatially inverted relative to a certain set of quantum fields, when those very fields are what ordinary matter is made of. Such a relative drag would not even make sense as a mathematical model. it would be ruled out, not because it fails to satisfy the equations of the theory, but because it is a contradiction in terms.

\section{Breaking mirroring as a special global sym- metry}

For simplicity, consider as in the beginning manifolds diffeomorphic to $\mathbb{R}^{4}$, so only one chart is needed. Let $g$ be the metric on $\mathcal{M}$ and let $\tau^{(\alpha)}, \alpha=0,1,2,3$ be a tetrad field on $\mathcal{M}$, a quadruple of vector fields $\tau^{(\mu)}: \mathcal{M} \rightarrow T\left(\mathbb{R}^{4}\right)$, $g\left(\tau^{(0)}, \tau^{(0)}\right)=1, g\left(\tau^{(0)}, \tau^{(j)}\right)=0, g\left(\tau^{(j)}, \tau^{(k)}\right)=-\delta^{j k}, j, k=1,2,3$. Let $r: \mathcal{M} \rightarrow \mathcal{M}$ satisfy

$$
\tau^{\mu}(q)=\left(2 \delta^{0 \mu}-1\right) \tau^{\mu}(r(q)), q \in \mathcal{M} .
$$

This manifold map induces global mirroring of the tetrad field. Evidently the latter can be used to define coordinates $x^{\mu}$ on $\mathcal{M}$ (so that $\tau^{(\mu)}=\partial / \partial x^{\mu}$ ), on which $r$ likewise induces global mirroring. In this way we define global mirroring for every tensor field on $\mathcal{M}$.

What about spinor fields? From our previous discussion (§3.2) there is a separate stipulation connecting the spin space $V_{q}$ at $q \in \mathcal{M}$ with the tangent space $T_{q}$, as given by (5). Let us indicate this by $\sigma$, with the opposite choice given by $\bar{\sigma}$. Let $F$ denote tensor fields and spin fields taken together, with the understanding that for spinor fields $r^{*} F$ is the induced point-wise transformation only (with no action on 'directions' in spin space, which are looked after by $\sigma$ ). Then a priori (trivial) mirror symmetry is the condition:

$$
\text { if }\langle\mathcal{M}, g, F, \sigma\rangle \text { is a model }\left\langle\mathcal{M}, r^{*} g, r^{*} F, \bar{\sigma}\right\rangle \text { is a model }
$$

or equivalently

$$
\text { if }\langle\mathcal{M}, g, F, \sigma\rangle \text {, is a model }\langle r(\mathcal{M}), g, F, \sigma\rangle \text { is a model }
$$


meaning, as before, that if the one is a model of a physically possible world then so is the other. This is the extension of the statement of a priori mirror symmetry to apply to spin-structures on manifolds.

Before considering the meaning of special mirror symmetry on such manifolds, with an eye to its violation, we should first consider how mirroring might be violated even in the absence of spin-structures. The obvious candidate for the expression of mirroring as a special symmetry is:

$$
\text { if }\langle\mathcal{M}, g, T\rangle \text { is a model }\left\langle\mathcal{M}, g, r^{*} T\right\rangle \text { is a model. }
$$

We earlier dismissed the failure of this condition as adequate for characterizing parity violation, as the latter is defined in a Minkowski space theory, but it does characterize a certain kind of mirror symmetry breaking: any theory in which mirroring is neither an isometry of $g$, nor of $T$, will do. A prime example is GTR.

That might seem a disappointment: GTR breaks special mirror symmetry with no need for chiral spinors; any asymmetric configuration of a single scalar field will do. But thinking again, the implication is welcome, for the failure of any other special symmetry $t$ in GTR is expressed as the absence of the associated isometry, $t^{*} g \neq g$; why suppose that mirroring should be any different? My claim throughout is that mirroring is to be treated no differently from any other symmetry. And anyway, unless a special symmetry is required by a theory, that theory should not be bound to respect it; GTR requires no special, continuous spacetime symmetry, so why should it require special mirror symmetry?

On the other hand, one speaks of certain models of GTR as satisfying a symmetry, rather than GTR itself-and, by extension, of certain models as violating a special symmetry. Thus Schwarzchild spacetime has spherical symmetry but not translational symmetry; Robertson-Walker spacetimes violate time translation symmetry (they are not static) but they are spatially homogeneous and isotropic. Many models have mirror symmetry in this sense.

Mirroring, we conclude, whether as a trivial or special symmetry, falls into place in GTR in exactly the same way as do continuous symmetries. The real difference is between GTR and other dynamical theories; between the inherent lack of symmetry of the distribution of fields on the manifold, as compared to the inherent symmetries of fields in the tangent space at each point of the manifold. This is the arena of all the non-gravitational physics, so it is no surprise that symmetry-breaking at this level has a fundamentally different character from that in GTR. It is sufficient therefore to consider only Minkowski space theories.

\subsection{Minkowski-space theories}

Theories on Minkowski space are of course theories on manifolds, so we may continue with the same notation, with the difference that the metric $g$ is fixed once and for all as that of Minkowski space, denoted $\eta$. It is homogeneous, isotropic, and mirror symmetric.

The condition for special mirror-symmetry breaking (SMB) is then: 
There is some model $\langle\mathcal{M}, \eta, T, \sigma\rangle$ such that $\left\langle\mathcal{M}, \eta, r^{*} T, \sigma\right\rangle$ is not a model

(SMB)

(and equivalently, by trivial mirror symmetry, that $\langle\mathcal{M}, g, T, \bar{\sigma}\rangle$ is not a model). In fact (SMB) would appear to be maximally violated in the presence of the electroweak interaction: for no model $\langle\mathcal{M}, g, T, \sigma\rangle$, is $\left\langle\mathcal{M}, g, r^{*} T, \sigma\right\rangle$ a model. And evidently what is ruled out is mathematically well-defined-there is no contradiction in reflecting (relatively dragging) only spinor fields at each point $q \in \mathcal{M}$, and not tensor fields as well. Rather, one ends up with relative configurations of fields in spacetime that do not satisfy the equations of QET.

But how are the two descriptions to be compared-how are the equations for the whole system to be set up, that make use of no external standard? Will they not also make use of a cross-product, as with (6a), (6b), or the equivalent choice of representation of the $\gamma$-matrices? Indeed, but we have already granted this point. By all means make use of a purely abstract standard for 'the left', say the left atlas of charts. The relationist claim is that any such abstract standard is ex hypothesis irrelevant to the distinguishing feature, whatever it is, that determines the standard for 'the left' within the model (the internal standard), the standard whose congruence and anticongruence relations with other handed bodies determines the use of all other chiral terms. Further, the relationist's presupposition is that any body of mathematics that can be built up using the left atlas can equally be built up using the right atlas-that the two structures will be a priori isomorphic. That is to say, by trivial mirror symmetry, either (6a) or (6b) can be used in setting up the total system of equations; it makes no difference. The total system of equations in either case will be isomorphic.

So far, and for the reasons mentioned at the beginning of $\S 3.2$, we have made no mention of Hilbert space structures or the quantum state. But trivial mirror symmetry has a reasonably clear expression in these terms: it is that, if $P$ is the unitary operator representing spatial inversion (in odd dimensions) on a Hilbert space $\mathcal{H}$, on which the Hamiltonian is the operator $H$, and the state $\Psi$ describes the total system, then the one system of equations is $\langle\mathcal{H}, H, \Psi\rangle$, whilst the other is $\left\langle\mathcal{H}, P H P^{-1}, P \Psi\right\rangle$. That makes the triviality of the claim perfectly obvious. But that also makes it clear that it is a mistake to suppose that parity violation amounts to the statement that whilst $\Psi(t)$ is a possible history of the closed system, $P \Psi(t)$ is not (for we have just granted that it is).

Returning to the system of fields, mirroring is a special symmetry if all of the spinor fields can be reflected, but none of the tensor fields. That would require that the spinor fields themselves carry a representation of the group of spatial inversions. We have already seen that this is not so in QET, although it is true of QED and quantum chromodynamics. But whilst that expresses the failure of mirroring as a special symmetry in QET, it does not give us all that we were promised. The suggestion was that we could find an internal, dynamical standard for our use of chiral terms, with lawlike congruence relations with other systems and fields; so far, it seems all that is on offer are aspects of the structure of QET itself. 


\subsection{Dynamical internal standards, again}

We are asking for something to fill the role of (MS2b), something that is naturally invariant under mirroring as an a priori symmetry, something that can actually be used. What is needed is a standard sensitive to the electroweak force, to which it couples directly, but which is at the same time observable or at least indirectly observable. That suggests the standard should be close to microscopic. Very simple examples at the ultra-microscopic would be different kinds of parity-violating decay processes-whose relative orientations could not be reversed consistent with dynamical laws-but much better would be a stable quantum system, not so large so as to unmanageably complicated, nor too small or too fleeting to function even in principle as an observable standard.

The obvious place to look is chiral chemistry. Indeed, very soon after the fall of parity, lawlike effects of this kind were conjectured. Thus Ulbricht (1959) argued that where $\beta$ rays could influence the decomposition of enantiomeric molecules, they would do so preferentially. Irradiation by radionuclides, he speculated, would produce differential decompositions of enantiomers in a racemic mixture, with one type more abundant than the other. It was the first in a long line of investigations into the influence of the electroweak force on the behaviour of atoms and molecules.

The effect conjectured by Ulbricht turned out to be negligible, but the contribution to the Hamiltonians of enantiomeric molecules due to the weak neutral current is not. The resulting differences in binding energies lead to observably different thermal reactions for these isomers (Rein 1974, Letokhov 1975). For the order of magnitude, the change in sign in the contribution to the total energy of an enantiomer depending on its relative orientation to the electroweak current is:

$$
\Delta E \approx \eta Z^{5} \times 10^{-19} \mathrm{eV}
$$

where $Z$ is the atomic number of the molecule and $\eta$ is the fractional dissymmetry factor (Zel'dovich et al 1977). In the example of Hegstrom et al. (1979), a triatomic sulphide chromophore C-S-C in the chiral field of other atoms of the steroid gives a fractional factor $\eta \approx 10^{-8}$. The same authors report much larger factors (of order $10^{-4}$ ) for certain ethylenes, where the chromophores are inherently monochiral.

Some took these results to show that the electroweak force explains the observed homochiral biochemistry of the Earth (as speculated by Mason and Tranter [1984]), rather than putting it down to a quirk of evolution. That would be icing on the cake. For our purposes, it is enough that the relative orientations that it produces with certain chiral molecules are lawlike. Thus in (MS2b), the mentioned enantiomers may be taken as, e.g., sulphide chromophores, in the lowest of the two ground states. The relative orientation of these, with the decay products of hyperon decay as depicted in Figure 3, is lawlike, and their relative inversion dynamically prohibited. This will do as an internal, dynamically embedded, standard of orientation. 
Still, this kind of internal determination, as specifying the relative orientation of something to something else, seems roundabout. Isn't it possible to shortcircuit this procedure, and just talk of the electroweak currents themselves, as the enantiomers mentioned in (MS2b)? It may be epistemically further removed, but the orientations of sulphide chromophores are hardly directly observable. Aren't we simply displaying ways in which the weak interaction manifests itself in molecular physics?

A similar objection can be made to our formulation of (SMB) in GTR: do not the matter fields and the metric field in this theory mesh so tightly that obviously they have the same orientation, if oriented at all? Why, to express mirror-symmetry breaking in GTR, do we have to speak of two fields here, and not one?

The answer is that there really do have to be two fields or structures or things, and not one, if my two central contentions are true-if mirroring is an a priori symmetry, and if relationism, in the guise of the invariance principle, applies to mirroring just as it does to other symmetries. Something handed has to be compared with something handed, if there is to be a relative orientation, itself invariant under mirroring, the only meaningful kind. As Weyl said, just before the discovery of parity violation:

Had God, rather than making first a left and then a right hand, started with a right hand and then formed another right hand, He would have changed the plan of the universe not in the first but in the second act, by bringing forth a hand which was equally rather than oppositely oriented to the first-created specimen. (Weyl 1952, $21-22)$.

His claim is just as true today.

\subsection{Accounting for contingency}

When Pauli quipped in the face of parity violation that 'God is weakly left handed', the implication, presumably, was that God could have been righthanded instead. What would the universe have been like in that case?

The easy answer is that whereas (1a) is empirically correct (referred to Figure 2 ) it could have been (1b) instead. But that is to treat 'left' and 'right' as external determinations. The hand depicted in Figure 2, an external standard, is by fiat dynamically decoupled from the electroweak fields, so of course it appears only a contingent matter that it has the relative orientation with respect to decay processes that it has. We have been over this ground already. As an account of how God could have differed in his designs, it is nothing but hubris. It is not that God had a distinctive handedness, which could have been different; it is that we humans have a distinctive handedness, that could have been different.

If there is anything to the idea that the fundamental forces could have had the opposite handedness to the one that they have, we need to consider lawlike relations between chiral fields. We should then consider the examples already 
given-where certain homochiral isomers are dynamically preferred over their enantiomers, and have such-and-such relative orientation to others. The alternative way that parity might have been broken would have led to the opposite relative orientation of all of those things.

But there is a great deal wrong with this way of putting it. It suggests that there is an identity of structure in the way things could have been after all so we are back to the kind of underdetermination that the invariance principle and LE were designed to circumvent. And when one looks at the concrete examples of a relative drag of such a standard, keeping others fixed, or keeping the geometry of certain electroweak decays fixed, it is an entirely open question whether there is any theory that could produce such a dynamics-and if there were one such theory, for a specified range of relative orientations, perhaps there are many, for that specified range.

Whence the stubborn intuition that there are exactly two possibilities? It derives, perhaps, from confusing physical distinctions with mathematical ones (the underlying a priori mirror symmetry). Or, more plausibly, it derives from confusing global and subsystem symmetries. As a subsystem symmetry, just because the standard used is not itself dynamically analyzed, it is by definition arbitrary. As a matter of logic there are exactly two possible ways in which the symmetry could be broken, with nothing to choose between them.

The better way of putting it, if we must speak in Pauli's terms, is that one of God's hands is weaker than the other-and that there are no further facts about God's anatomy that distinguish the two. That makes clearer that there are not two possible worlds he could have created (but infinitely many, depending on how much weaker the one hand is than the other).

\subsection{Orientation fields}

In recent literature on mirror symmetry and parity violation the suggestion has often been made that an orientation field is needed (Wald 1984, Huggett (2000)), and that otherwise parity violation is non-locally determined (Hoefer (2000), Pooley (2003)).

An orientation field serves a simple intuition. Imagine a parity-violating decay takes place in one region of spacetime, and that another, similar, decay takes place elsewhere, at large spacelike separation. What guarantees that the two processes are handed in the same way? It had better be something local to each of the two regions of spacetime, if we are not to have brute distant correlations; so there must be a locally available standard, varying continuously from point to point, which directs the dynamics and guarantees (assuming the manifold is orientable) that the sense of the decay process is everywhere the same.

But opinions differ on what such a field could be. Huggett (2000) pursued an aspect of the absolute-relational controversy that we have been ignoring, namely, the question of whether inertial or affine structure is available to a relationist who denies the reality of space or spacetime, and, if not, what should be put in its place (essentially, the question of how to implement a Machian 
dynamical theory). Newtonian principles pick out a certain class of preferred states, namely, inertial motions: this needed a new structure to underpin it, an affine connection. Parity-violating theories likewise pick out a certain class of preferred states, namely those in which parity is violated with a certain sense: this too needs some new structure to underpin it. The suggested candidate is an 'orientation field'.

In illustration, Huggett (2000) wrote down a simple 1-dimensional 2-particle quantum mechanical potential function (with $\lambda$ and $\mu$ positive real numbers):

$$
V\left(x_{1}, x_{2}\right)=\lambda\left(x_{1}-x_{2}\right)+\mu\left(x_{1}-x_{2}\right)^{2}=V_{A}+V_{S}
$$

where by construction $V_{A}$ is odd and $V_{S}$ is even under mirroring. If the particles have the same mass, $m$, the Schrödinger equation is:

$$
\frac{\hbar^{2}}{2 m}\left(-\nabla_{1}^{2}-\nabla_{2}^{2}+V_{A}+V_{S}\right) \Psi=i \frac{\partial \Psi}{\partial t} .
$$

It explicitly violates mirror-symmetry. The result is that. e.g., initially symmetric (even) states evolve into superpositions of even and odd states. The equation drives a 2 -particle state, initially with a uniform probability density over a given segment $S \times S$ of $\mathbb{R}^{2}$, into one concentrated in a region where $x_{1}-x_{2}$ is negative. If the negative real line extends to the left, and the positive to the right, particle 1 will, if found in $S$, be more probably found to the left of particle 2 .

Huggett claims that the distinction between $x_{1}<x_{2}$ and $x_{1}>x_{2}$, on which the potential depends, has to be given

In absolute terms, not just relative to some arbitrary coordinates. Thus, not until an 'arrow of space' is given is the theory well-defined. This arrow can tell us for two points whether their separation is positive or negative-which is the 'earlier' spatially speaking-and hence give definite meaning to the Hamiltonian of the theory. (Note the analogy with Newton's first law: 'constant motion', and thus the law, is ill defined unless some notion of affine structure is given.) Of course, once we have observed the development of the particles we could determine the direction of the arrow, and could express its direction in relational terms, say by two standard objects and their order. Once again, the relationist is not faced with a descriptive problem-or even an epistemological problem-but with formulating a theory of the process in suitable relational terms, and a plausible theory should not make fundamental reference to a contingent standard. (Huggett 2000, p.235).

An orientation field, an 'arrow to space', is by contrast more suitable.

There are points of overlap between us. Huggett's complaint about contingent standards is the same as mine. We agree that the standard should be internal to the model; but more than that, it should not be any old handed object; it should not be contingent. He should welcome, then, my suggestion 
that we choose certain chiral molecules instead. But this sits unhappily with his comparison of the orientation field to an affine structure: chiral chromophores are not exactly fundamental in that sense. Nor do they have to be specified in advance to define the dynamics, on the contrary, they are manifestations of the dynamics.

The better way of reading Huggett's remark from our point of view is that the choice of an 'arrow of space' is to be understood as the choice of the leftatlas, say, rather than the right-atlas. We may grant that only then is the theory well-defined (only then is it written down in a definite system of coordinates); grant too that the choice of the atlas of charts (whether the right-atlas or the left) should be regarded as endowing the manifold with a particular structure (call it an orientation field rather than a choice of atlas); allow that only thus is the theory properly formulated in 'plausible' and 'suitable' relational terms. But what does not follow is that we have two distinct theories-on the contrary, our claim is that the choice of an orientation field in this sense, whether the left atlas or the right atlas, makes no difference to the physics.

But Huggett insists there are two distinct theories here, depending on the choice of the arrow to space, a view backed up by Pooley:

The orientation field is either supposed to be a real, physical field, or is supposed to represent some genuinely asymmetric structure of space or spacetime itself. If this is the case, then one cannot identify a theory that assigns a certain probability to the vector from particle 1 to particle 2 being aligned with the arrow defined by the orientation field, with a theory that assigns precisely that probability to the case where the two vectors are in the opposite alignment. Similarly, a theory that asserts that all electrons which are 'congruent' to an orientation field couple to $W$ bosons and those which are 'incongruent' do not, cannot be identified with a theory that predicts the same phenomena by asserting that all electrons which are incongruent to an orientation field couple to $W$ bosons. (Pooley 2003, 273-4).

Given Pooley's presuppositions that the orientation field is a real physical field or a genuinely asymmetric structure of space-if the orientation field enters into real (invariant) dynamical relations with other fields-then assuredly the two relative alignments of the fields would be physically distinct. Pooley's example of two such distinct alignments-between fermion fields and bosonic (tensor) fields, and oppositely oriented fermion fields with the same couplings to the same boson fields-is also similar to ours. But we are saying that where one is possible (satisfies the equations of QET) the other is not. The molecule with the mirror-image spatial geometry of the sulphur chromophore in its lowest energy ground-state, leaving all else unchanged, is not in its lowest energy ground-state. A fortiori, if one had two theories which differed in this respect they would not be the same.

Huggett and Pooley on the contrary see in (7) two distinct theories which are observationally the same. Why think that? One reason is because one 
thinks that if mirror symmetry is broken, it must be because one of exactly two possible worlds (mirror-images of each other) is singled out as real-whereupon there must be another theory that picks out the other world as real-but that, as good relationists, the two worlds do not differ observationally. This fits with the thought, already discussed, that if $\Psi$ is a solution to a parity-violating Hamiltonian $H$, then $P \Psi$ presents the mirror-inverted world, and is prohibited (where $U$ is spatial inversion).

To this our answer is as before: there is only one theory here, with two isomorphic representations, the one $\langle\mathcal{H}, H, \Psi\rangle$, the other $\left\langle\mathcal{H}, P H P^{-1}, P \Psi\right\rangle$. Or it might be better said that this is a parity-violating theory only when referred to an external standard, where mirroring is broken as a subsystem symmetry. Indeed it describes only a lone, 2-particle hand, in 1-dimensional space, with God's creative decision awaiting the next step, the creation of a second, handed system.

If there is no natural candidate 'orientation field' in our framework that gives Huggett and Pooley all that they want, what of its original rational-to define a local internal standard? The orientation field was to tell, in each local vicinity, which way particles like hyperons in that vicinity were to decay; otherwise there would only be 'the relationist's account of parity violation, with its brute, lawlike non-localities' (Pooley 2003, 274).

The choice of right-atlas (respectively left-atlas) obviously does not serve this function, for the left-atlas (respectively right-atlas) will reproduce exactly the same physics. But there is another candidate, surely, which does, namely the entire system of electroweak fields. The fields, we suppose, must be in place and have the structure that they have - whatever their quantum excitation numbers-throughout all of spacetime. The fields do not pop in and out of existence when particles do.

To be sure, we are back to controversial questions about how, precisely, a relativistic quantum field theory is to be interpreted. In response to this, we could stick to classical field theory. It is true that classical fields (unlike quantum fields) can be strictly zero throughout an open set, and that may suggest they cannot be relied on to furnish a local standard; but this surely leads to much more general questions-for example, of whether the local Lagrangian function is so much as defined in a region in which the fields of which it is a function disappear.

But even if there is a problem with locality arising in this way, it is far from clear that it is specific to parity-violation. We know that not all goes well in this respect for gauge theories more generally, classical as well as quantum. The Aharonov-Bohm effect is a case in point. Here the interference fringe produced by an electron beam is displaced, depending on the value of the magnetic flux through a solenoid in the region of the interfering beams. The values of the electromagnetic potentials themselves are not real, by the invariance principle, whereas invariant scalars constructed from the $\vec{E}$ and $\vec{B}$ fields all vanish in the region dynamically accessible to the electrons, the region outside the solenoid. The effect, from the point of view of invariant local quantities, is holistic, to be 
described in terms of the holonomy of the field-invariant quantities associated with closed curves encircling the solenoid. Neither is this effect a purely quantum one; similarly, in GTR, on parallel transport along a curve encircling a cone, there is a holonomy effect, depending on the curvature at the tip of the cone, despite the fact that everywhere along the curve the metric is flat.

\subsection{Conclusions}

Relationism is commonly thought to take away the reality of space, and in the context of handedness, to take away the reality of 'left' and 'right'. But neither conclusion follows from the invariance principle alone.

In the context of handedness, there are well-known arguments to show that the concepts 'left' and 'right', if they do have meaning, are ineffableincommunicable-and dependent on ostension. Since relationists, rightly, deny that 'left' and 'right' differ as intrinsic geometric determinations, there is the temptation to agree that whatever meaning they have is bound up with ostension, and is nonconceptual. But that temptation should be resisted.

The most famous of these arguments is posed by way of a problem, Gardner's (1964, ch. 18) Ozma problem: 'suppose you are in contact with an extragalactic civilization, location unknown: to communicate the meaning of 'left' by a language transmitted in the form of pulsating signals'. To this relationists typically agree the problem is insoluble, because to convey what we mean by 'left' to someone remote from us is to establish extrinsic spatial relations (congruence or anticongruence) between us and them-and that cannot be done by specifying information only about the relata. This is mixed in with the general view, traceable to Kant, that 'left' and 'right', in practise, are learned by ostension, so cannot be taught from afar, without a common object to which each party can ostensively refer.

But putting things this way, though not exactly wrong, make facts about mirroring look more mysterious than they really are. The role of ostension in language use, essential as it is, brings in questions of much greater scope, that apply to shape recognition (and much else) quite generally: there need be no special difficulty with the term 'left'. And there is a simple solution to the Ozma problem on the table: the meaning of 'left' lies in such-and-such facts about chiral chemistry, and various relative congruences between enantiomeric molecules and other dynamically specified bodies. Knowing the meaning of left, and all there is to know about QET, one knows the relative orientation of every electroweak decay process. All of this can be conveyed in the equations, without ever having to know which of the right atlas, or the left atlas, is to be used (for either can be used).

But do I know, by these methods, which of my hands, fingers stretched, is congruent to which dynamically described monochiral molecule? No, not by these methods-supposing there are in fact no law-like relations between human anatomy and chiral chemistry. If their relation is purely contingent, nothing short of observation, ostensive comparison, will do. But given this observation and the information thus obtained: can I convey it to my distant friends? But 
of course I can-for it is the information that the normal human heart is on the side of the hand that is congruent to certain dynamically-specified molecules. And do they, in that other galaxy, thereupon have to observe objects in common with us-presumably the same chiral chemistry-to know which hand that is? But why should observation come into it, supposing they know everything there is to know about chiral chemistry (as determined by the equations)? They know which dynamically-specified molecule we are talking about. And they know that their local chiral chemistry (insofar as it is anchored to the weak interaction) is the same as ours (for it is the same everywhere)-that is secured by the nature of field theory, as just discussed.

But they may have another question whose answer they want to know namely, which of their appendages matches most closely that hand of ours. That is equivalent to the question, with our signal to them received and understood, as to which of their appendages is congruent to which monochiral molecule. To answer that, assuming their gross anatomy is no more constrained by law-like relations to chiral chemistry than ours, they will have to make observations of their own. Thus there are two observations, one in each galaxy, each concerning contingencies of different anatomies: but neither telling us what 'left' really is, for that has already been dynamically characterized.

\section{Acknowledgments}

My thanks to a number of audiences, too numerous to mention, who have heard me speak on various of these ideas or their precursors, and for helpful discussions over the years to Harvey Brown, Nick Huggett, Oliver Pooley, David Wallace, and Graeme Segal, none of whom may entirely agree with my conclusions. My special thanks to Oliver Pooley for convincing me of the comparative irrelevance of PCT symmetry to the treatment of mirroring.

\section{References}

Bartlett, S., T. Rudolph, and R. Spekkens (2005), 'Reference frames, superselection rules, and quantum information', quant-ph/0610030.

Brown, H. (2005), Physical Relativity. Oxford: Clarendon Press.

Brown, H., and R. Sypel, (1986), 'On the meaning of the relativity principle and other symmetries', International Studies in the Philosophy of Science 9: 235-53.

Crawford, F., M. Cresti, M. Good, K. Gottstein, E. Lyman, F. Solmitz, M. Stevenson, and H. Ticko, (1957), 'Detection of parity nonconservation in $\Lambda$ decay', Physical Review 108: 1102-3.

Davis, W. (1970), Classical Fields, Particles, and the Theory of Relativity. New York: Gordon and Breach.

Earman, J. (1989), World Enough and Space-Time. Cambridge, MA: MIT Press.

Earman, J., and J. Norton (1987), 'What Price Substantivalism? The Hole Story', British journal for the Philosophy of Science 38: 515-25. 
Gardner, M. (1964), The Ambidextrous Universe: Mirror Asymmetry and TimeReversed Worlds. New York: Basic Books.

Hegstrom, R., D. Rein, and P. Sanders, (1979), 'Parity Non-conserving Energy Difference between Mirror Image Molecules', Physics Letters A 71: 499.

Hoefer, C. (2000), 'Kant's Hands and Earman's Pions: Chirality Arguments for the Substantival Space', International Studies in the Philosophy of Science 14: $237-56$.

Huggett, N. (1999), Space from Zeno to Einstein: Classic Readings with a Contemporary Commentary. Cambridge, M.A: MIT Press.

Huggett, N. (2000), 'Reflections on parity non-conversation', Philosophy of Science 67: 219-41.

Letokhov, V. S. (1975), 'On Difference of Energy Levels of Left and Right Molecules Due to Weak Interactions', Physics Letters A 53: 275.

Mason, S. and G. Tranter (1985), 'The Electroweak Origin of Biomolecular Handedness', Proceedings Royal Society London A 397: 45-65.

Nozick, R. (2001), Invariances: The Structure of the Objective World. Cambridge, MA: Harvard University Press.

Peskin, M. and D. Schroeder (1995), An Introduction to Quantum Field Theory. Reading, MA: Addison-Wesley.

Pooley, O. (2003), 'Handedness, Parity Violation, and the Reality of Space', in K. Brading and E. Castellani (eds.), Symmetries in Physics: Philosophical Reflections. Cambridge: Cambridge University Press, 250-280.

Rein, D. (1974), 'Some Remarks on Parity Violating Effects of Intramolecular Interactions', Journal Molecular Evolution 4: 15.

Saunders, S. (2003) 'Indiscernibles, General Covariance, and Other Symmetries: the Case for Non-Reductive Relationalism', in A. Ashtekar, D. Howard, J. Renn, S. Sarkar, and A. Shimony, (eds.), Revisiting the Foundations of Relativistic Physics: Festschrift in Honour of John Stachel. Dordrecht: Kluwer, 151-175.

Stachel, J. (1993), 'The Meaning of General Covariance', in A. Janis, N. Rescher, and G. Massey, (eds.), Philosophical Problems of the Internal and External Worlds: Essays Concerning the Philosophy of Adolf Grünbaum. Pittsburgh: University of Pittsburgh Press.

Ulbricht, T. L. V. (1959), 'Asymmetry: The Nonconservation of Parity and Optical Activity', Quarterly Reviews (London) 13: 48-60.

Wald, R. (1984), General Relativity. Chicago: Chicago University Press.

Weyl, H. (1952), Symmetries. Princeton: Princeton University Press.

Zel'dovich, B., D. Saakyan, and I. Sobel'man (1977), 'Energy Difference between Right-Hand and Left-Hand Molecules, Due to Parity Nonconservation in Weak Interactions of Electrons with Nuclei', Soviet Physics JETP Letters 25: 94-97. 